\title{
Efficacy of a Ready-to-Drink Gelled Water and of a Thickening Powder in Patients with Oropharyngeal Dysphagia: a Crossover Randomized Study
}

\author{
Jean-Yves Salle ${ }^{1}$ - Achille Tchalla ${ }^{2} \cdot$ Remy Thirion $^{3} \cdot$ Annick Offret $^{3,4} \cdot$ Laurence Dussaulx $^{5} \cdot$ Florence Trivin $^{5}$. \\ Caroline Gayot $^{2}$ - Philippe Fayemendy ${ }^{6,7,8}$. Pierre Jésus ${ }^{6,7,8}$. Cécile Bonhomme ${ }^{9}$ Etienne Hazart ${ }^{10}$. \\ Charlotte Baudry $^{10}$ - Jean-Claude Desport ${ }^{6,7,8}$
}

Accepted: 18 February 2021 / Published online: 22 July 2021

(C) The Author(s) 2021

\begin{abstract}
Management of oropharyngeal dysphagia (OD) is mainly based on modifying liquid viscosity and solid consistency in order to preserve oral feeding while avoiding unsafe swallowing. Adding thickening powders (TP) to water is the most common practice in patients suffering from OD to liquids, but ready-to-use gelled waters (RGW) can also be proposed. The main objective of this study was to assess the efficacy of a RGW and a TP on swallowing in hospitalized patients with different OD etiologies. This open, crossover, multicenter trial recruited thirty hospitalized patients with OD to liquids, confirmed by positive 3-ounce water test or positive Practical Aspiration Screening Scheme test. The patient's ability to swallow $120 \mathrm{~g}$ of a RGW (IDDSI level 4) and a drink prepared with TP (nectar viscosity; NTP, 291 cP, IDDSI level 2; or if necessary, honey; HTP, $769 \mathrm{cP}$, IDDSI level 3) was evaluated in a random order at 1- to 3-day intervals. The main criterion was the efficacy of each product, defined as the proportion of patients who successfully swallowed without immediate reflexive cough. The RGW and TP were successfully swallowed in respectively $93.3 \%$ (95\% CI: 77.999.2) and 82.8\% (95\% CI: 64.2-94.2) of patients with different dysphagia etiologies (stroke, neurodegenerative diseases, or aging) and unable to swallow thin water. Taste and texture of both study products were well appreciated by patients, with a preference for the RGW over TP. Therefore, the use of these thickened products could be part of the therapeutic strategy for patients with OD to liquids.
\end{abstract}

Keywords Dysphagia $\cdot$ Swallowing $\cdot$ Thickened liquids $\cdot$ Viscosity $\cdot$ Thickening powder $\cdot$ Ready-to-drink gelled water

\section{Introduction}

Oropharyngeal dysphagia (OD), a prevalent condition recognized by the World Health Organization (WHO) in the

This article is part of the Topical Collection on Medicine

Etienne Hazart

etienne.hazart@fr.lactalis.com

Charlotte Baudry

charlotte.baudry@lactalis.fr

1 Service de Médecine Physique et Réadaptation, Hôpital Jean Rebeyrol, Avenue du buisson, 87042 Limoges cedex, France

2 Service de Médecine gériatrique, CHU Dupuytren, 2, Avenue Martin Luther King, 87042 Limoges cedex, France

3 Service de Gériatrie, CH René Pleven, 74, Rue Châteaubriand, 22100 Dinan, France
International Classification of Diseases [1], is characterized by difficulties in swallowing liquids and/or solids that may lead to aspiration. OD must be rapidly diagnosed and managed in order to prevent related severe complications

4 Service de Neurologie, CH Broussais, 1, rue de la Marne, 35400 Saint Malo, France

5 Service de Réadaptation digestive et nutritionnelle, Clinique St Yves, 4, rue Adolphe Leray CS 54 435, 35044 Rennes Cedex, France

6 Unité de Nutrition et CSO du Limousin, CHU Dupuytren, 2, Avenue Martin Luther King, 87042 Limoges cedex, France

7 Réseau LINUT, 16, rue du Cluzeau, 87170 Isle, France

8 Faculté de Médecine, Inserm UMR 1094 NET, rue du Dr Marcland, 87032 Limoges cedex, France

9 Lactalis Nutrition Santé, Parc d'activités Secteur Est, 35370 Torcé, France

10 Lactalis R\&D, 8, Fromy, 35240 Retiers, France 
(aspiration, undernutrition, dehydration, anxiety related to meals and social isolation) [2]. OD prevalence is high in the elderly, after stroke, in neurodegenerative diseases, and in head and neck cancer patients [2]. Management of OD is mainly based on modifying liquid viscosity and solid texture/consistency in order to preserve oral feeding while avoiding unsafe swallowing [3]. A well-established intervention in hospitals for patients with OD is to thicken liquids by adding thickeners in water as described in previous studies [4-6]. Prescription of thickness level is patient-specific. Different viscosity levels were defined for liquids in 2002 by the National Dysphagia Diet (NDD) task force [7] according to their rheological properties: nectar thick liquid (51-350 cP), honey thick liquid (351-1750 cP), and spoon/pudding thick liquid (>1750 cP). More recently in 2017, the International Dysphagia Diet Standardization Initiative (IDDSI) proposed new definitions for texture-modified foods and thickened liquids for OD [8]. A five-level scale of drink thickness has been established from thin (level 0 ) to extremely thick liquids (level 4) $[8]$.

Gum-based thickeners are able to thicken a wide range of liquids at different temperatures and maintain stable viscosity over time [9]. However, adding thickening powder to liquids can be constraining for caregivers. That is why ready-to-drink gelled waters (RGW) have been developed, formulated with pectin and gums to ensure their stability during transport and storage in hospitals. Both gum-based thickeners and RGW are widely used for dysphagic patients and seem to be well accepted [9]. There are many commercially available products with different components and rheological properties, but none has been clinically studied to prove its efficacy in current hospital practices. Videofluoroscopy (VFS) is considered the "gold standard" method to assess the effect of increased viscosity on swallowing [9] and is largely used to evaluate thickened drink. However, this method does not apply to RGW that are solid gelled water, because stirring for the addition of contrast product needed for such evaluation would induce a modification of the texture and viscosity level of RGW. Similarly, viscosity measurement by rheology would destroy the gel structure and would modify its texture. Finally, considering that mastication and hydration processes of the bolus are not reproducible by technical evaluation [10], a clinical evaluation of the safety of products is required.

This is the first study to assess the effectiveness on swallowing of two commercial products, a RGW and a thickening powder (TP,) in standard hospital practices. Therefore, the main objective of this study was to assess the efficacy of the consumption of an entire cup of RGW, or the same amount of thickened water $(120 \mathrm{ml})$, in hospitalized patients with different OD etiologies.

\section{Methods}

\section{Patients}

Patients with OD hospitalized in acute or rehabilitation hospitals in France, for whom aspiration was detected, were prospectively included. Inclusion criteria were age $>18$ years, suffering from OD following neurological diseases (stroke or neurodegenerative diseases) or aging, positive 3-ounce water test (3oz WT) or positive Practical Aspiration Screening Scheme (PASS) test, and able of understanding and signing written consent after receiving study information. The PASS test combines the Clinical Predictive Scale of Aspiration (CPSA) and the $3 \mathrm{oz}$ WT in patients falling in the uncertain range of CPSA (between 14 and 28) [11]. The CPSA is a noningestion test consisting of a questionnaire with six clinical signs correlating with aspiration risk. The $3 \mathrm{oz}$ WT was performed for all the patients at inclusion and was considered positive when it evidenced coughing after ingestion of $90 \mathrm{ml}$ of water [12]. Non-inclusion criteria were medical or surgical history which interfered with the participation to the study, intolerance to one or more components of the products, and diabetic patient. All participants provided written informed consent. The protocol (registration number: 2014-A0108443) was approved by French authorities: the Agence Nationale de Sécurité du Médicament and the Comité de Protection des Personnes Sud-Ouest et Outre-Mer IV, Limoges, France. The trial was conducted according to the principles and rules of the Declaration of Helsinki and its subsequent amendments.

\section{Products and Design of the Study}

Three grenadine-flavored thickened waters were studied: one RGW and two drinks prepared with TP at two different viscosities. Compositions of study products are detailed in Table 1. The RGW (Gelodiet, Delical, Lactalis Nutrition Santé, France) was a 120-g cup (IDDSI level 4). The two other drinks were prepared with a TP (Gelodiet, Delical, Lactalis Nutrition Santé, France) as followed: at room temperature, $8.4 \mathrm{~g}$ grenadine syrup was mixed with either $5.5 \mathrm{~g}$ of TP and mineral water (qsp $120 \mathrm{~g}$ ) to obtain nectar viscosity (NTP, 291 cP, IDDSI level 2) or $6.9 \mathrm{~g}$ of TP to obtain honey viscosity (HTP, 769 cP, IDDSI level 3).

After positive 3oz WT at the inclusion visit, patients were randomized in a crossover, open study to assess the efficacy of swallowing of the products following the sequence "RGW then NTP" or "NTP then RGW." Randomization was done by automatic list generation. The different tests were performed at 1- to 3-day intervals.

The patient's ability to swallow $120 \mathrm{~g}$ of each product was evaluated. The product was considered effective if it did not induce an immediate reflexive cough or the 
Table 1 Products composition and viscosity for $120 \mathrm{~g}$

\begin{tabular}{llll}
\hline & RGW & NTP & HTP \\
\hline Energy (kcal) & 37 & $19\left(53^{*}\right)$ & $24\left(57^{*}\right)$ \\
Protein (g) & 0.1 & 0.0 & 0.0 \\
Fat (g) & 0 & 0 & 0 \\
Carbohydrates $(\mathrm{g})$ & 8.5 & $4.4\left(12.8^{*}\right)$ & $5.5\left(13.9^{*}\right)$ \\
Fibers (g) & 1.2 & 0.7 & 0.9 \\
Sodium (mg) & $\mathrm{NS}$ & 9.3 & 11.6 \\
Calcium (mg) & 23 & 0 & 0 \\
Chlorine (mg) & 52 & 0 & 0 \\
Mean viscosity (cP) & $\mathrm{NE}$ & 291 & 769 \\
NDD viscosity range & $\mathrm{NA}$ & Nectar $(51-350 \mathrm{cP})$ & Honey $(351-1750 \mathrm{cP})$ \\
IDDSI level & 4 & 2 & 3 \\
\hline
\end{tabular}

$R G W$ ready-to-drink gelled water, NTP nectar-like thickened powder, HTP honey-like thickened powder. NDD National Dysphagia Diet (7), IDDSI International Dysphagia Diet Standardization Initiative (8), NS not specified, $N E$ not evaluable, $N A$ not applicable

*Values with syrup inability to swallow leading to split out the bolus. If the NTP induced a reflexive cough, a subsequent test with the HTP was performed at the same visit. Total amount of liquid consumed and test duration were recorded. Patients stayed under medical observation for $1 \mathrm{~h}$ after ingestion of each study product. The main criterion of the study was the efficacy of each product, defined as the proportion of patients who successfully swallowed without immediate reflexive cough.

A satisfaction questionnaire was completed at the end of each intake test. Appreciation of taste and texture as well as global appreciation of each product was scored using a visual scale based on seven smiley levels as previously described [13]. Patients were also questioned about their intended consumption of each product and the amount of thickened/gelled water they would be ready to consume daily. Any adverse event occurring during the study was recorded, assessed for severity and related or not with product intake according to the investigator's opinion.

\section{Statistical Analysis}

Statistical analyses were performed, using JMP® 13.0 from SAS Institute (Cary, USA). Quantitative variables were described by mean \pm standard deviation (SD) and compared using the Student $t$-test for paired data or the non-parametric Wilcoxon test. The main criterion was efficacy of each product. The $95 \%$ confidence intervals were calculated for each proportion using the ClopperPearson method. Efficacies of the two products (RGW and TP) were compared a posteriori using a McNemar test. Results were considered significant when $p$-value $<0.05$.

\section{Results}

Five centers with different specialties participated to the trial from September 2015 to September 2016: one Physical and Rehabilitation Medicine Service, two Geriatric Services, one Neurology Unit, and one Digestive and Nutritional Rehabilitation Service. The flow chart of the study is given in Fig. 1. Thirty hospitalized patients with OD were included in the study. Characteristics of the study population are presented in Table 2. CPSA was evaluated for 28 patients, and $86 \%$ of them were at potential risk of aspiration (score $\leq 28$ ). All the patients had a positive $3 \mathrm{oz}$ WT at inclusion. Ninetythree percent of the patients had been placed on a thickened fluid recommendation prior to inclusion, and two patients (7\%) were not orally hydrated (complementary enteral feeding).

RGW was tested by all patients ( $n=30)$ and TP by 29 patients because one patient refused to consume the product. Twenty-seven patients were assessed with the NTP in agreement with the protocol. The two patients who were not orally hydrated were directly assessed with the HTP because of a higher risk of aspiration according to the investigator's opinion. Total amount of thickened liquid consumed during the test and test duration was similar between the RGW and the TP (Table 3). Seventy-three percent of patients entirely consumed each study product. Two patients on 30 coughed after ingestion of RGW. Five patients on 29 coughed after ingestion of TP; three coughed after both NTP and HTP, and two did not test NTP and coughed after HTP. The two patients who coughed after RGW also presented impaired swallowing with TP. The two patients who were not orally hydrated coughed after HTP, but one of them successfully swallowed RGW. Finally, RGW was successfully swallowed in $93.3 \%$ (95\% CI: 77.9-99.2) of patients and TP in $82.8 \%$ (95\% CI: 
Table 2 Characteristics of the study population at inclusion

$n(\%)$ or mean $\pm \mathrm{SD}$

Sex

\begin{tabular}{|c|c|}
\hline $\begin{array}{l}\text { Men } \\
\text { Women }\end{array}$ & $\begin{array}{l}15(50) \\
15(50)\end{array}$ \\
\hline Age (years) & $74.3 \pm 13.5$ \\
\hline \multicolumn{2}{|l|}{ Age distribution } \\
\hline $\begin{array}{l}\circ \leq 60 \text { years } \\
\circ 60-80 \text { years } \\
\circ \geq 80 \text { years }\end{array}$ & $\begin{array}{l}5(17) \\
13(43) \\
12(40)\end{array}$ \\
\hline Body mass index $\left(\mathrm{kg} / \mathrm{m}^{2}\right)$ & $25.1 \pm 4.3$ \\
\hline \multicolumn{2}{|l|}{ Etiology of dysphagia } \\
\hline $\begin{array}{l}\text { Stroke } \\
\circ \text { Ischemic } \\
\circ \text { Hemorrhagic } \\
\text { Neurodegenerative diseases } \\
\circ \text { Parkinson's disease } \\
\circ \text { Autoimmune limbic encephalitis } \\
\circ \text { Cerebella degeneration by genetic atrophy } \\
\circ \text { Progressive supranuclear palsy } \\
\circ \text { Multiple sclerosis } \\
\text { Age-related dysphagia }\end{array}$ & $\begin{array}{l}20(67) \\
18(90) \\
2(10) \\
7(23) \\
3(43) \\
1(14) \\
1(14) \\
1(14) \\
1(14) \\
3(10)\end{array}$ \\
\hline CPSA score & $23.1 \pm 5.6$ \\
\hline \multicolumn{2}{|l|}{ CPSA score distribution } \\
\hline $\begin{array}{l}\circ<14 \text { (risk of aspiration) } \\
\circ 14-28 \text { (uncertain range) } \\
\circ>28 \text { (no risk) }\end{array}$ & $\begin{array}{l}2(7) \\
22(79) \\
4(14)\end{array}$ \\
\hline Positive 3oz WT (\%) & $30(100)$ \\
\hline
\end{tabular}

CPSA Clinical Predictive Scale of Aspiration, 3oz WT 3-ounce water test

64.2-94.2) of patients (Table 3). Only one non-serious adverse event was recorded related to the study product: difficulty to catch the breath during the test with RGW, resolved by a brief oxygen supply.

Results of the satisfaction questionnaire are presented in Table 4. Significant differences were observed between the two products for taste, texture, and global appreciation, indicating a preference for the RGW over TP (Table 4). However, no difference was observed for intended consumption of RGW or TP, with about $60 \%$ of patients who declared to be ready to consume each product daily (Table 4).

\section{Discussion}

This is the first study conducted using normal French hospital practices to treat dysphagic patients and based on the entire consumption of a glass of thickened water or a cup of ready to drink gelled water.

Our results showed that both products were effective to ameliorate swallowing in patients with OD from different etiologies. None of the 30 patients enrolled in this study was able to swallow thin liquid at inclusion, whereas $93 \%$ of them successfully swallowed RGW and $83 \%$ successfully swallowed TP. These results confirm that modifying liquid viscosity by adding thickening agents is an effective strategy for the management of OD.

Previous studies in patients suffering from OD showed an improvement of swallowing, defined as a reduced prevalence of penetration and aspiration evaluated by VFS or FEES (fiberoptic endoscopic evaluation of swallowing) [9]. Whatever the thickening agents used, thickened fluids were successfully swallowed by $50-100 \%$ of patients with OD [4-6, 9, 14, 15]. However, most of these studies were performed in patients including $20-60 \%$ who were still able to safely swallow thin liquids [4, 6, 9, 14, 15]. By contrast, in our study, all the patients demonstrated signs of aspiration and/or penetration on thin liquids and presented a reflexive cough after $90 \mathrm{ml}$ water ingestion at inclusion. Only the study of Leder et al. [5] enrolled patients who exhibited aspiration with thin liquid on FEES and showed that $100 \%$ of them successfully swallowed both nectar-like and honey-like thickened liquids. However, only 5-7 ml bolus volumes were tested, whereas aspiration could potentially occur with larger volumes. Moreover, Miles et al. [16] showed a significant association between cough response to aspiration and viscosity and volume of drink intake, but some of their patients coughed when they aspirated thin fluids but silently aspirated thick fluids indicating that cough response could be poor indicator of aspiration. However, the inversed reaction was observed within $7 \%$ of their patients ingesting a $5 \mathrm{ml}$ bolus and $1.7 \%$ of them ingesting a $50 \mathrm{ml}$ bolus. In our study, the ingested bolus was $120 \mathrm{ml}$. Indeed, increasing bolus volume was reported to significantly reduce the safety of swallow whatever the viscosity [9]. We choose to test a high quantity of drinks to be more representative of the common practice in hospital, and this may also reduce the risk of inobservance of silent aspiration

Despite their different viscosities and their different thickener agents, efficacy was not statistically different between the 2 tested products. However, RGW (IDDSI level 4) was successfully swallowed in three patients who coughed after NTP (IDDSI level 2) and/or HTP (IDDSI level 3) ingestion. This observation is consistent with the previous results showing that prevalence of safe swallowing significantly increased in a bolus viscosity-dependent manner, with maximal therapeutic effect observed with spoon-thick viscosity [4, 9, 14]. However, spoon-thick viscosity with modified starch thickeners has been reported to increase the prevalence of oropharyngeal residue, which may result in post-swallow airway ingestion [14]. In our study, the TP was tested only at nectar or honey viscosities and not at spoon-thick viscosity. RGW was more viscous than TP according to the IDSSI classification, but the NDD classification is not applicable to RGW because rheological measurements are not possible on this product. Presence of oropharyngeal residue was not evaluated in our 
Fig. 1 Flow chart of the study population. $R G W$ ready-to-drink gelled water, $T P$ thickened powder. The asterisk indicates one missing data

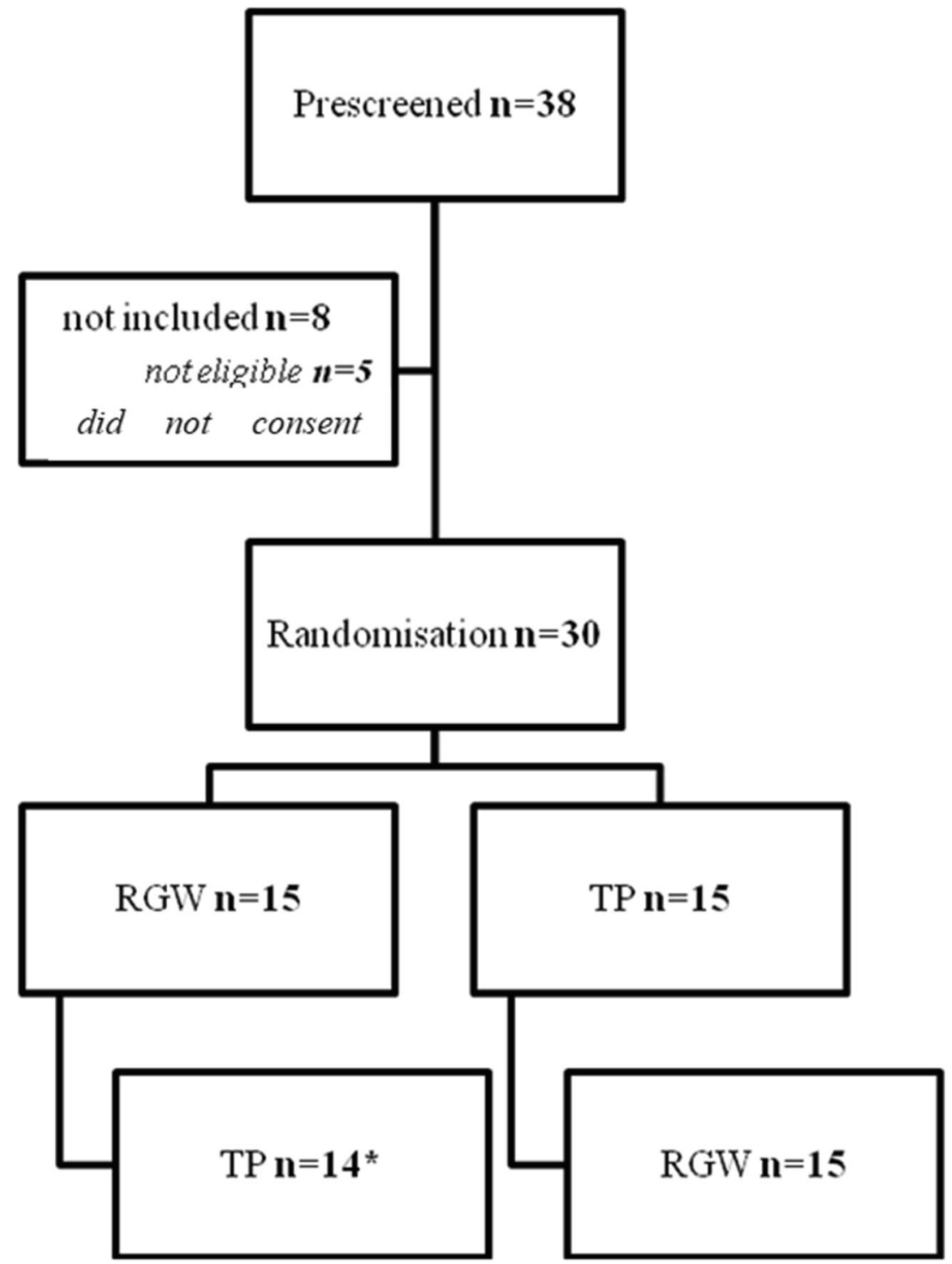

study, but patients remained under medical examination for $1 \mathrm{~h}$ after the ingestion test, in order to identify potential postswallow reactions.

Another important point in the management of OD is palatability of thickened fluids, because a good compliance is required to ensure an adequate level of hydration of patients.
An aversion for thickened liquids is often described in patients with OD, who declared to prefer nectar viscosity over honey or spoon-thick viscosities [17]. Therefore, it is recommended to reach a proper level of safety with the minimum amount of thickeners possible [18]. In our study, both products were well appreciated (rated above average) by the patients, with a

Table 3 Efficacy of the study products on swallowing

\begin{tabular}{lll}
\hline & RGW (n=30) & TP (n=29) \\
\hline Total amount of product consumed, g (\%) & $97.8(82)$ & $100.8(84)$ \\
Time needed to consume the study product, min (mean \pm & $5.6 \pm 3.2$ & $6.8 \pm 4.7$ \\
SD) & 2 & 5 \\
Number of patients who coughed after ingestion & $93.3(\mathrm{IC} 95 \%:$ & $82.8($ IC95 \%: \\
Efficacy (proportion of patients who successfully & $81.7-99.9)$ & $64.2-94.2)$ \\
$\quad$ swallowed, \%) & & \\
\hline
\end{tabular}

$R G W$ ready-to-drink gelled water, $T P$ thickened powder 
Table 4 Subjective appreciation of the two study products and intended use

\begin{tabular}{llll}
\hline & RGW $(n=28)$ & TP $(n=28)$ & $p$-value \\
\hline Appreciation of the product (from $0=$ bad to 7 = good), mean score \pm SD & & \\
Taste appreciation & $5.8 \pm 1.5$ & $4.6 \pm 1.8$ & 0.0008 \\
Texture appreciation & $5.3 \pm 1.5$ & $4.4 \pm 1.6$ & 0.01 \\
Global appreciation & $5.5 \pm 1.6$ & $4.4 \pm 1.7$ & 0.004 \\
Ready to consume the product daily, $n(\%)$ & $18(64)$ & $17(61)$ & $>0.05$ \\
If ready to consume the product daily, number of cups $(120 \mathrm{ml})$ per day; $n(\%)$ & \\
$<5$ cups/day & $13(72)$ & $8(47)$ & $>0.05$ \\
$5-8$ cups/day & $4(22)$ & $6(35)$ & \\
$\geq 9$ cups/day & $1(6)$ & $3(18)$ & \\
\hline
\end{tabular}

$R G W$ ready-to-drink gelled water, $T P$ thickened powder preference for RGW over TP. However, the RGW was specifically formulated to have optimal taste and texture, while TP-based drinks were obtained by mixing water, TP, and grenadine syrup, which may explain this difference. Around $60 \%$ of patients declared to be ready to consume RGW or TP daily, but most of them would be ready to consume less than 8 cups (i.e., $960 \mathrm{ml}$ ) per day, which is less than the recommended standards of fluid intake for hospitalized adults established at $1500 \mathrm{ml}$ [19]. Previous studies showed that patients consuming pre-thickened drinks or drinks thickened with TP consumed less than their recommended daily fluid intake orally $[20,21]$. Therefore, particular attention must be paid to avoid dehydration of dysphagic patients, and the use of thickened fluids needs to be completed by foods with high water content or use of non-oral supplementary routes [19,21].

There are several limitations in this study. First, sample size of 30 patients was low and did not include patients suffering from head or neck cancer, while prevalence of OD is high in this population. Clinical evaluation of efficacy of product on swallowing was based on the presence or absence of a reflexive cough after ingestion, and "silent aspiration" was not evaluated in this study. VFS is the gold standard method to evaluate deglutition but is not applicable with pre-thickened drinks. Further evaluation with the use of FEES and additional clinical parameters (such as voice changes and measurement of oropharyngeal residue) would be needed to complete our results and better evaluate the efficacy of the 2 tested products. However, the efficacy as measured in the present study was an easy-tomeasure non-invasive parameter that did not require specific material or trained staff and compatible with common hospital practice, but also that is realized with a high quantity of tested product to secure the cough response. All patients exhibited reflexive cough after water ingestion at inclusion, and our study showed that this specific symptom was significantly improved with thickened liquids.

\section{Conclusion}

This study showed that a RGW and a TP were successfully swallowed in $93 \%$ and $83 \%$, respectively, of hospitalized patients with different dysphagia etiologies and unable to swallow thin water. Therefore, the use of these thickened products could be part as the therapeutic strategy to improve patient safety and compliance as well as to contribute to the maintenance of adequate fluid intake to prevent dehydration.

Abbreviations $3 o z W T, 3$-ounce water test; CPSA, Clinical Predictive Scale of Aspiration; FEES, fiberoptic endoscopic evaluation of swallowing; HTP, honey-like thickened powder; IDDSI, International Dysphagia Diet Standardization Initiative; $N D D$, National Dysphagia Diet; $N T P$, nectar-like thickened powder; $R G W$, ready-to-drink gelled water; $T P$, thickening powder; $V F S$, videofluoroscopy

Acknowledgements We gratefully thank SLB Pharma (Rennes, France) for its advices and support for the management of this study and analysis of the results.

Author Contribution JYS, AT, RT, AO, LD, FT, CG, PF, PJ, and JCD make substantial contributions to acquisition, analysis, and interpretation of data. JCD participates in drafting the article. All authors participate in revising the article critically for important intellectual content. All authors give final approval of the version to be submitted and any revised version.

Funding This study was supported by the Lactalis group. The funding source had no influence in subjects' recruitment, data collection, and analysis.

Data Availability The authors confirm that the data supporting the findings of this study are available within the article.

\section{Declarations}

Ethics Approval The protocol (registration number: 2014-A01084-43) was approved by French authorities: the Agence Nationale de Sécurite du Médicament and the Comité de Protection des Personnes Sud-Ouest et Outre-Mer IV, Limoges, France. The trial was conducted according to the principles and rules of the Declaration of Helsinki and its subsequent amendments. 
Informed Consent All participants recruited were able to understand and sign written consent after receiving study information and provided written informed consent.

Conflict of Interest Cécile Bonhomme, Etienne Hazart, and Charlotte Baudry are employees of the Lactalis Group. Jean-Claude Desport participated in 2017 at the AFDN congress invited by Lactalis and was supported for training by Nutricia, in 2018 and 2019. None of the other authors has conflicts of interest to declare.

Open Access This article is licensed under a Creative Commons Attribution 4.0 International License, which permits use, sharing, adaptation, distribution and reproduction in any medium or format, as long as you give appropriate credit to the original author(s) and the source, provide a link to the Creative Commons licence, and indicate if changes were made. The images or other third party material in this article are included in the article's Creative Commons licence, unless indicated otherwise in a credit line to the material. If material is not included in the article's Creative Commons licence and your intended use is not permitted by statutory regulation or exceeds the permitted use, you will need to obtain permission directly from the copyright holder. To view a copy of this licence, visit http://creativecommons.org/licenses/by/4.0/.

\section{References}

1. WHO | International Classification of Diseases, 11th Revision (ICD-11). WHO. 2018.Available at https://www.who.int/ classifications/icd/revision/en/. accessed 26 October 2018.

2. Malagelada JR, Bazzoli F, Boeckxstaens G, De Looze D, Fried M, Kahrilas P, et al. World gastroenterology organisation global guidelines: dysphagia-global guidelines and cascades update September 2014. J Clin Gastroenterol. 2015;49(5):370-8.

3. Wirth R, Dziewas R, Beck AM, Clave P, Hamdy S, Heppner HJ, et al. Oropharyngeal dysphagia in older persons - from pathophysiology to adequate intervention: a review and summary of an international expert meeting. Clin Interv Aging. 2016;11:189-208.

4. Rofes L, Arreola V, Mukherjee R, Swanson J, Clave P. The effects of a xanthan gum-based thickener on the swallowing function of patients with dysphagia. Aliment Pharmacol Ther. 2014;39(10): 1169-79.

5. Leder SB, Judson BL, Sliwinski E, Madson L. Promoting safe swallowing when puree is swallowed without aspiration but thin liquid is aspirated: nectar is enough. Dysphagia. 2013;28(1):58-62.

6. Logemann JA, Gensler G, Robbins J, Lindblad AS, Brandt D, Hind JA, et al. A randomized study of three interventions for aspiration of thin liquids in patients with dementia or Parkinson's disease. J Speech Lang Hear Res. 2008;51(1):173-83.
7. National Dysphagia Diet Task Force. National Dysphagia Diet: Standardization for Optimal Care. American Dietetic Association, 2002.

8. Cichero JA, Lam P, Steele CM, Hanson B, Chen J, Dantas RO, et al. Development of international terminology and definitions for texture-modified foods and thickened fluids used in dysphagia management: the IDDSI framework. Dysphagia. 2017;32(2):293314.

9. Newman R, Vilardell N, Clave P, Speyer R. Effect of bolus viscosity on the safety and efficacy of swallowing and the kinematics of the swallow response in patients with oropharyngeal dysphagia: white paper by the European Society for Swallowing Disorders (ESSD). Dysphagia. 2016;31(2):232-49.

10. Vallons KJR, Oudhuis L, Helmens HJ, Kistemaker C. The effect of oral processing on the viscosity of thickened drinks for patients with dysphagia. Ann Rehabil Med. 2015;39(5):772-7.

11. Zhou Z, Salle J, Daviet J, Stuit A, Nguyen C. Combined approach in bedside assessment of aspiration risk post stroke: PASS. Eur J Phys Rehabil Med. 2011;47(3):441-6.

12. DePippo KL, Holas MA, Reding MJ. Validation of the 3-oz water swallow test for aspiration following stroke. Arch Neurol. 1992;49(12):1259-61.

13. Maitre I, Symoneaux R, Sulmont-Rossé C. 23-Sensory testing in new product development: working with older people. Rapid Sensory Profiling Tech. 2015:485-508.

14. Vilardell N, Rofes L, Arreola V, Speyer R, Clave P. A comparative study between modified starch and xanthan gum thickeners in poststroke oropharyngeal dysphagia. Dysphagia. 2016;31(2):169-79.

15. Clave P, de Kraa M, Arreola V, Girvent M, Farre R, Palomera E, et al. The effect of bolus viscosity on swallowing function in neurogenic dysphagia. Aliment Pharmacol Ther. 2006;24(9):1385-94.

16. Miles A, McFarlane M, Scott S, Hunting A. Cough response to aspiration in thin and thick fluids during FEES in hospitalized inpatients. Int J Lang Commun Disord. 2018;53(5):909-18.

17. Garcia JM, Chambers E, Matta Z, Clark M. Viscosity measurements of nectar- and honey-thick liquids: product, liquid, and time comparisons. Dysphagia. 2005;20(4):325-35.

18. Cichero JA. Thickening agents used for dysphagia management: effect on bioavailability of water, medication and feelings of satiety. Nutr J. 2013;12:54.

19. Murray J, Miller M, Doeltgen S, Scholten I. Intake of thickened liquids by hospitalized adults with dysphagia after stroke. Int $\mathrm{J}$ Speech Lang Pathol. 2014;16(5):486-94.

20. Whelan K. Inadequate fluid intakes in dysphagic acute stroke. Clin Nutr. 2001;20(5):423-8.

21. Patch CS, Tapsell LC, Mason S, Curcio-Borg F. Thickened fluids: factors affecting wastage. Int J Speech Lang Pathol. 2003;5(2):737.

Publisher's Note Springer Nature remains neutral with regard to jurisdictional claims in published maps and institutional affiliations. 